patient. Each centre has a close association with a university.

Not all the patients in the region can expect to be treated in the clinical units, for many highly competent doctors may not be involved or may be unwilling to be associated with co-operative activities. Coercion to attempt to make established practitioners change their habits is doomed to failure. On the other hand if adequately supported clinical units can show that it is under these circumstances that cancer can be treated more effectively, they will be their own advertisement and more of their colleagues will put aside their initial doubts, fears, and prejudices and offer their services to enhance the co-ordinated effort of the centre. The oncological centres, by fostering the relations between clinicians and scientists and providing a common meeting ground, should go far in overcoming difficulties in collaboration that have hampered cancer research in the past.

\section{Ischaemia of the Intestinal Tract}

The intestine receives its blood supply from three anastomotic channels-the coeliac artery and the superior and inferior mesenteric arteries. So rich is this source and its connexions that one or even two of these trunks can become gradually occluded without the development of any demonstrable pathological lesion. However, impaired arterial flow may result in one of three syndromes-intestinal infarction, chronic intestinal ischaemia, and ischaemic enteritis and colitis.

Acute mesenteric vascular occlusion may be either arterial or venous. In B. B. Jackson's collected series of 1,500 cases $62 \%$ were arterial, $33 \%$ venous, and $5 \%$ mixed. ${ }^{1}$ Of the arterial occlusions $42 \%$ were embolic (the great majority secondary to auricular fibrillation), $38 \%$ secondary to arteriosclerotic thrombosis, and in $20 \%$ no organic obstruction to major vessels was found. In this last group there is a high association with intractable congestive cardiac failure. ${ }^{2}$ In elderly arteriosclerotic patients partial occlusion of the superior mesenteric artery is common, and if mesenteric perfusion is still further reduced as a result of hypotension due to shock, anaemia, or myocardial failure intestinal ischaemia may supervene.

The commonest features of mesenteric infarction are the sudden onset of acute abdominal pain, shock, vomiting, distension, and diarrhoea. Stools and vomit often contain blood. The patient is usually beyond middle age and there is frequently a history of previous cardiac disease. Some patients have symptoms which suggest previous abdominal angina. Examination shows a normal or lowered temperature, a rapidly rising pulse with falling blood pressure, pallor, abdominal tenderness, progressive distension, but relatively little guarding. ${ }^{3}$ Leucocytosis of 20,000 or more is common. In an ageing population this emergency is being seen with increasing frequency. $H$. Droller ${ }^{4}$ has recently reported 13 cases of vascular disease of the coeliac axis and mesenteric vessels in 1,700 geriatric patients admitted to St. James's Hospital, Leeds.

Until recently the only possible surgical procedure was resection of the infarcted intestine, first reported by $\mathrm{J} . \mathbb{W}$. Elliott in 1895.5 The mortality for this procedure remains high, especially when extensive segments of gut are involved. A major advance was made by A. A. Klass, ${ }^{6}$ of Winnipeg, who reported two cases of superior mesenteric artery embolectomy which he performed in 1951 and 1953. Though both the patients died, later experience from many centres has shown that embolectomy may be successful with complete or partial salvage of the ischaemic bowel. A. Marston ${ }^{7}$ points out that direct attack on the origin of the superior mesenteric artery is difficult; it is easier to expose the ileocolic artery, through which a Fogarty catheter may be passed upwards to the origin of the superior mesenteric artery. Embolus or thrombus is extraoted, and if the peripheral circulation to the gut is fully restored the ileo-colic artery can then be safely ligated. If, however, there is still doubt about the blood flow, a side-to-side anastomosis can be performed between the ileo-colic and the right common iliac artery to revascularize the bowel in a retrograde fashion.

It must be emphasized that prognosis, even today, remains grave, with a montality in the region of 80 to $90 \%$. A patient who survives a massive resection after infarction may present serious metabolic problems. Usually when more than $75 \%$ of the intestine is lost the "short bowel" syndrome results. It may be possible to improve absorption in severe cases by fashioning an antiperistaltic reversed intestinal segment, placed at the lower end of the remaining small intestine, to delay passage of bowel contents and thus increase the absorptive power of the remaining gut segment. ${ }^{8}$

Chronic intestinal ischaemia may result in "intestinal angina"-a syndrome of pain which comes on shortly after meals, weight loss, and diarrhoea. Physical signs are absent, though an epigastric bruit may be detected. Aortography is required to demonstrate the arterial occlusion, but it must be stressed that the mere presence of atheroma in the visceral vessels by no means indicates that symptoms are due to ischaemia, since occlusions are common in symptomless patients. A. P. Dick and his colleagues ${ }^{9}$ studied 100 consecutive patients who had undergone aortography. There were 27 control patients with no clinical evidence of arterial disease who had been investigated for abdominal masses and other conditions, 56 hypertensive patients undergoing renal aortography, 12 with peripheral arterial disease, and a fourth group of five patients with chronic intestinal ischaemia. A significant difference was found between the means of total cross-sectional areas of the coeliac, and superior and inferior mesenteric arteries in the cases with ischaemic gut disease and in all other groups, and between that in the patients with arterial disease and the controls. However, there was considerable overlap of individual cases. Reconstructive surgery at present should be advised only with great caution and after other causes of abdominal pain, both organic and functional, have been excluded.

The third group of patients comprise those with ischaemic disease insufficient to produce frank necrosis of the bowel but in whom the specialized tissue-the mucosa and muscleundergo ischaemic damage. This may be transient and followed by complete or partial healing, or may be followed by mucosal ulceration and fibrotic replacement of muscle. This phenomenon is especially likely to affect the splenic flexure of the colon, giving rise to the syndrome of "ischaemic colitis" which was first described and classified by Marston and his colleagues ${ }^{10}$ in 1966 . They defined three groups: ischaemic colitis with gangrene, ischaemic stricture, and transient ischaemic colitis. The ischaemic origin of this condition has been confirmed both by experimental studies in the $\operatorname{dog}^{11}$ and by the observation by D. C. Rowe-Jones ${ }^{12}$ of the development of a typical stricture at the splenic flexure in a patient who has been found to have a colonic infarct at 
laparotomy but who was too ill to undergo resection at that time.

R. W. Marcuson and J. A. Farman ${ }^{13}$ have now been able to review 122 collected cases of ischaemic disease of the colon. Of these, 98 were over the age of 50, and of the 24 younger patients three were diabetic and six were women taking oral contraceptives. There were 103 cases of ischaemic colitis and 16 examples of gangrene of the colon. All sites in the large bowel were involved, including the rectum, but 71 of the cases involved the transverse colon, splenic flexure, or descending colon. Unless there is evidence of spreading peritonitis, in which case surgery must be undertaken, the authors advise that all cases should be treated conservatively. Nearly half will resolve and require no further treatment. The remainder will develop stricture, but even some of these may dilate as they mature, and surgery is indicated only for obstruction or when malignant disease cannot be excluded.

\footnotetext{
1 Jackson, B. B., Occlusion of Superior Mesenteric Artery. Springfield,

2 Berger, R. L., and Byrne, J. J., Surgery, Gynaecology, and Obstetrics, 1961, 112, 529

3 Ellis, H., in Abdominal Operations, ed. R. Maingot, 5th edn., 2, 1497. New York, Appleton-Century-Crofts, 1969.

4 Droller, H., Age and Ageing, 1972, 1, 162.

5 Elliott, J. W., Annals of Surgery, 1895, 21, 9.

- Klass, A. A., Fournal of the International College of Surgeons, 1953, 20, 687. Marston, A., Annals of the Royal College of Surgeons of England, 1972, 50, 29.

${ }^{8}$ Venables, C. W., Ellis, H., and Smith, A. D. M., Lancet, 1966, 2, 1390.

'Dick, A. P., Graff, R., Gregg, D. Mc. G., Peters, N., and Sarner, M., Gut, 1967, 8, 206 .

10 Marston, A., Pheils, M. T., Thomas, M. L., and Morson, B. C., Gut, 1966, 7, 1.

11 Marston, A., Marcuson, R. W., Chapman, M., and Arthur, J. F., Gut, $1969,10,121$.

13 Rowe-Jones, C. D., British Medical fournal, 1969, $1,361$.

13 Marcuson, R. W., and Farman, J. A., Proceedings of the Royal Society of Medicine, 1971, 64, 1080 .
}

\section{Tragic Dilemma}

A recent case in the Hull juvenile court was concerned with principles that have been the subject of much anxious debate among doctors for some time. No transcript of evidence was taken, but from press reports ${ }^{1} 2$ the circumstances of the case appear to be as follows. A baby boy of 13 months suffered from spina bifida and hydrocephalus. In the opinion of medical consultants the baby needed surgical treatment to save his life. The parents refused their consent to operation, and the director of social services, acting under the Hull Social Services Committee, therefore applied to the court for the child to be taken into the care of the local authority. The court made the required order, and after sitting in private the social services committee gave its consent to the operation.

There can be no suggestion that anyone here had any but the most scrupulous regard for the child's welfare. The law was observed in the spirit and the letter, and it is clear that all parties in the case acted from the highest motives. But in this tragic dilemma of what was best for the baby the parents and committee found themselves in irreconcilable opposition.

In a press interview with the parents ${ }^{3}$ the mother was reported to have said:

"We love our son like any other parents, but we don't want him to lie in hospital suffering operation after operation and be used as a guinea-pig. There is no religion involved in this. We would be overjoyed if a miracle happened and he became well again. The terrible truth is that he will never walk, no matter how many operations he has. If he falls over while sitting up in bed, he remains in that position until a nurse pushes him back again. It was heartbreaking enough to learn that he was handicapped, without doctors prolonging our agony. This dreadful dilemma could befall any family at any time and they might find themselves getting the rubber stamp treatment. If courts and doctors are going to decide about life, it won't be long before they are sitting in judgment about death."

The view of the social services committee was given in the following statement ${ }^{1}$ :

"We had regard on the one hand to the fact that the parents had refused to give their consent to the operation and, on the other, the opinion of the medical consultants that it is essential that the child should have this operation. The local authority has a clear duty to have regard to the welfare of the child in its widest sense and we are satisfied that in view of what the medical consultants have said about this case that it will be in the child's best interests for him to undergo this operation."

Local authorities are rarely called upon to seek statutory powers to remove a child from its parents' control so that it may have medical treatment to which the parents object. The use of these powers is certainly something the medical profession should scrutinize closely, for it is ultimately on a medical man's authority that the law intervenes to sever the closest of all human bonds, between parent and child. Medical decisions with such painful implications are rarely likely to be simple to reach, and one feature of any case that must provoke the most careful thought is the expected results of treatment. When a child is sick and can be restored to normal health by treatment, the advice a doctor should give is much clearer than when the proposed treatment will not restore the child to normal health. These are matters that can be judged only in relation to each individual case. The problem of what treatment-if any, in some circumsstances-should be given to babies with gross congenital defects ${ }^{4}$ can be difficult enough for a doctor to offer parents frank advice on when he is confident that they are likely to accept it. When he knows they do not agree with him the burden of decision is all the heavier.

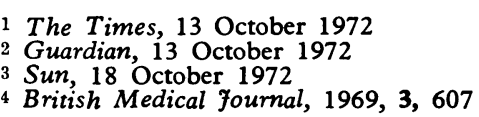

\section{Corticosteroids in Pulmonary Aspergillosis}

Clear examples of immunopathological mechanisms in allergic disease of the lungs are provided by the different clinical disorders caused by Aspergillus fumigatus, which can grow in the lungs. ${ }^{1}$ Analysis of the allergic factors shows the place of corticosteroids in treatment.

In atopic patients the spores act as allergens, stimulating the production of reaginic (IgE) antibody, which mediates immediate type I asthmatic reactions. Disodium cromoglycate inhibits these reactions, ${ }^{2}$ whereas corticosteroids do not, ${ }^{3}$ nor do they modify appreciably the immediate skin test reaction. The production in these patients of precipitins capable of mediating type III immune-complex reactions is attributed to the growth of the spores in the bronchi. A. fumigatus is cultured more frequently from the sputum of asthmatic than of non-asthmatic people. ${ }^{4}$ The presence together of the types I and III allergy is closely associated with the com- 